\title{
Sequential product spaces are Jordan algebras
}

\author{
Cite as: J. Math. Phys. 60, 062201 (2019); doi: 10.1063/1.5093504 \\ Submitted: 21 February 2019 - Accepted: 12 May 2019 • \\ Published Online: 10 June 2019

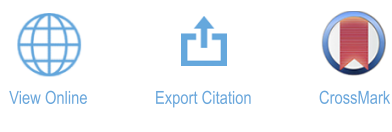

John van de Wetering

AFFILIATIONS
Institute for Computing and Information Sciences, Radboud University, Toernooiveld 212, Nijmegen, Netherlands

a) Electronic mail: john@vdwetering.name

\begin{abstract}
We show that finite-dimensional order unit spaces equipped with a continuous sequential product as defined by Gudder and Greechie are homogeneous and self-dual. As a consequence of the Koecher-Vinberg theorem, these spaces therefore correspond to Euclidean Jordan algebras. We remark on the significance of this result in the context of reconstructions of quantum theory. In particular, we show that sequential product spaces must be $\mathrm{C}^{*}$-algebras when their vector space tensor product is also a sequential product space (in the parlance of operational theories, when the space "allows a local composite"). We also show that sequential product spaces in infinite dimension correspond to JBalgebras when a few additional conditions are satisfied. Finally, we remark on how changing the axioms of the sequential product might lead to a new characterization of homogeneous cones.
\end{abstract}

Published under license by AIP Publishing. https://doi.org/10.1063/1.5093504

\section{INTRODUCTION}

The set of observables of a quantum system can be represented as the space of self-adjoint operators on a complex Hilbert space $B(H)^{\mathrm{sa}}$. This space has a variety of algebralike structures that can be associated with it, the most well-known of which is the Jordan product $a * b:=\frac{1}{2}(a b+b a)$. In the 1930s, Jordan, von Neumann, and Wigner hoped to find generalizations of the quantum mechanical formalism by considering general spaces equipped with an axiomatization of this algebraic structure. They however found that the resulting Euclidean Jordan algebras (EJAs) have a strikingly simple classification ${ }^{1}$ and hence that this algebraic approach does not allow you to go far beyond quantum theory. The significance of EJAs was further established by the Koecher-Vinberg theorem that states that any finite-dimensional homogeneous and self-dual ordered vector space is a Euclidean Jordan algebra. ${ }^{2}$ These two results, the Koecher-Vinberg theorem and the classification by Jordan, von Neumann, and Wigner, lie at the heart of many reconstructions of quantum theory where intuitively sensible axioms from which quantum theory can be derived are sought ${ }^{3-7}$ (although it should be noted that these theorems are not used directly in all approaches $^{8-10}$ ).

Other algebraic structures on $B(H)^{s a}$ studied in an axiomatic way are the quadratic Jordan algebras that axiomatize the map $(a, b) \mapsto$ $a b a$ or the more general triple product $(a, b, c) \mapsto \frac{1}{2}(a b c+c b a)$. The definitions of the Jordan product and the triple product do not have a particularly compelling physical motivation: the product does not correspond to any type of physical process. In this paper, we will look at a different structure that does follow naturally from physical processes. Given two positive operators $a$ and $b$, we define their sequential product as $a \& b:=\sqrt{a} b \sqrt{a}$. When $a$ and $b$ represent effects, i.e., possible outcomes in a measurement, then the sequential product models the act of first getting the outcome $a$ and then the outcome $b$, hence the name sequential product (although this composition is also known as a Lüders process). It is important to note that this product is only defined for positive operators (since otherwise the square root would not be defined) and that this operation is not bilinear, associative, or commutative. Gudder and Greechie introduced the concept of a sequential effect algebra to study the sequential product in a more abstract setting. ${ }^{11}$ While they studied the structure of the sequential product on the very general structure of effect algebras, we will restrict ourselves to the more concrete setting of order unit spaces:

Definition 1. An order unit space $(V, \leq, 1)$ is an ordered real vector space with the additional property that 1 is a strong Archimedean unit:

1. Strong unit: For all $a \in V$, we can find $n \in \mathbb{N}$ such that $-n 1 \leq a \leq n$.

2. Archimedean: For $a \in V$, when $a \leq \frac{1}{n} 1$ for all $n \in \mathbb{N}_{>0}$, then $a \leq 0$. 
We call the elements $a \in V$ with $0 \leq a \leq 1$ the effects of $V$ which we will denote by $[0,1]_{V}$. The states of $V$ are positive linear maps $\omega: V \rightarrow \mathbb{R}$ such that $\omega(1)=1$.

Ordered vector spaces with a strong unit represent the most general kinds of systems allowed in causal generalized probabilistic theories, ${ }^{12}$ and hence, they form a suitable background to studying models of general physical theories. The Archimedity condition states intuitively that there are no effects that cannot be distinguished by a state. More precisely, order unit spaces are precisely the ordered vector spaces where the states order-separate the elements: if $\omega(v) \leq \omega(w)$ for all states $\omega$, then $v \leq w$.

Note that an order unit space has a norm induced by the order in the following way: $\|a\|:=\inf \left\{r \in \mathbb{R}_{\geq 0} ;-r 1 \leq a \leq r 1\right\}$. Whenever we refer to continuity in the context of order unit spaces, it should be understood to refer to this norm.

The object of study in this paper is an order unit space with an operation modeled after the sequential product on $B(H)^{\text {sa }}$. To be specific:

Definition 2. Let $(V, \leq, 1, \&)$ be an order unit space equipped with a binary operation $\&:[0,1]_{V} \times[0,1]_{V} \rightarrow[0,1]_{V}$. We write $a \mid b$ and say $a$ and $b$ are compatible when $a \& b=b \& a$. We call $V$ a sequential product space and \& a sequential product when $\&$ satisfies the following properties for all $a, b, c \in[0,1]_{V}$ :

(S1) Additivity: $a \&(b+c)=a \& b+a \& c$.

(S2) Continuity: The map $a \mapsto a \& b$ is continuous in the norm.

(S3) Unitality: $1 \& a=a$.

(S4) Compatibility of orthogonal effects: If $a \& b=0$, then also $b \& a=0$.

(S5) Associativity of compatible effects: If $a \mid b$, then $a \&(b \& c)=(a \& b) \& c$.

(S6) Additivity of compatible effects: If $a \mid b$, then $a \mid 1-b$, and if also $a \mid c$, then $a \mid(b+c)$.

(S7) Multiplicativity of compatible effects: If $a \mid b$ and $a \mid c$, then $a \mid(b \& c)$.

The properties we require of $\&$ are the same as that of a sequential product in a sequential effect algebra ${ }^{11}$ except for condition S2 which is new. It should be noted that the standard sequential product $a \& b=\sqrt{a} b \sqrt{a}$ on $B(H)^{\mathrm{sa}}$ is not fully characterized by these axioms, as there are multiple binary operations that satisfy these axioms. ${ }^{13}$ It is possible however to characterize the standard sequential product using related sets of axioms. ${ }^{14-16}$ The main examples of sequential product spaces are Euclidean Jordan algebras:

Definition 3. We call a real vector space $V$ a Jordan algebra when it has a bilinear commutative operation $*$ that satisfies the Jordan identity: $a *(b *(a * a))=(a * b) *(a * a)$. We call $V$ a Euclidean Jordan algebra (EJA) when it is furthermore a finite-dimensional Hilbert space with inner product $\langle\cdot, \cdot\rangle$ such that $\langle a * b, c\rangle=\langle b, a * c\rangle$.

Euclidean Jordan algebras were originally introduced as generalizations of the quantum mechanical formalism under the name formally real Jordan algebras. ${ }^{17}$ Due to their connection to self-dual homogeneous cones, EJAs have been studied in many different contexts as well. The main examples of Euclidean Jordan algebras are the sets of self-adjoint operators $B(H)^{\mathrm{sa}}$, where $H$ is a real, complex, or quaternionic Hilbert space where the Jordan product is given by $A * B:=\frac{1}{2}(A B+B A)$. In fact, as shown by the classification theorem of EJAs, ${ }^{1}$ any EJA can be constructed as a direct sum of these algebras, a family of algebras called spin-factors, and the exceptional algebra.

It is not obvious how EJAs form sequential product spaces. Using the quadratic map, which is in turn defined in terms of the Jordan product, a sequential product can be constructed. ${ }^{16}$ The main purpose of this paper is to establish that EJAs are in fact the most general type of (finite-dimensional) sequential product space:

Theorem. Let $V$ be a finite-dimensional sequential product space. Then, $V$ is order-isomorphic to a Euclidean Jordan algebra.

This result is given as Theorem 1 in Sec. III D. Since EJAs are very well understood and, in particular, classified, we can use this theorem to prove additional results. In particular, using a property called local tomography we can infer when sequential product spaces are $\mathrm{C}^{*}$-algebras.

Definition 4. Let $V$ and $W$ be finite-dimensional sequential product spaces. We say that they have a locally tomographic composite when their vector space tensor product $V \otimes W$ is also a sequential product space with $\left(a_{1} \otimes b_{1}\right) \&\left(a_{2} \otimes b_{2}\right)=\left(a_{1} \& a_{2}\right) \otimes\left(b_{1} \& b_{2}\right)$ for all effects $a_{i}$ in $V$ and $b_{i}$ in $W$.

In the context of generalized probabilistic theories, the property of local tomography states that local measurements on each of the subsystems are enough to fully characterize bipartite states. It is a property that holds for regular quantum theory but fails for, for instance, quantum theory over the real numbers.

Theorem. Let $V$ be a finite-dimensional sequential product space that has a locally tomographic composite with itself. There exists a $C^{*}$-algebra $A$ such that $V$ is isomorphic to $A^{\text {sa }}$ as a Jordan algebra. 
This result is given as Theorem 3 in Sec. V. Note that $\mathrm{C}^{*}$-algebras being singled out among all the EJAs by local tomography is not surprising as similar results were obtained in Refs. 5 and 18-20, but in combination with the results regarding the sequential product, it does give a novel understanding of the mathematical structure of quantum theory:

"A causal probabilistic physical theory that satisfies local tomography and that has a well-behaved notion of sequential measurement must be modeled by $\mathrm{C}^{*}$-algebras.”

There have been quite a few characterizations of quantum theory using operational axioms, ${ }^{4-6,8-10,19,21}$ but the one presented above is different in a couple of ways. First of all, other characterizations and reconstructions have their axioms refer to a multitude of structures, like the existence of certain systems, transformations, and pure states, instead of focusing on a single aspect (in this case, sequential measurement). Second, all reconstructions of quantum theory that the author is aware of have axioms ensuring the existence of suitable reversible (i.e., invertible) dynamics in the theory. By contrast, this characterization of $\mathrm{C}^{*}$-algebras does not directly say anything about the existence of reversible maps.

The proof we use for the correspondence between sequential product spaces and EJAs is highly specific to finite-dimensional spaces. Yet, by assuming a few other properties, we can also find a correspondence between $\sigma$-directed complete sequential product spaces and JB-algebras, which form an infinite-dimensional generalization of Euclidean Jordan algebras. We refer to Sec. VI for details.

The structure of the paper is as follows: the main theorem that finite-dimensional sequential product spaces are EJAs will be proved using the Koecher-Vinberg theorem which requires us to show that the space is both homogeneous and self-dual. In Sec. II, we will cover the known results originally presented in Refs. 11 and 16 regarding sequential product spaces, culminating in a proof of a spectral theorem and a proof of the homogeneity of the space. Then, in Sec. III, we will prove self-duality of the space, using results regarding the lattices of projections of Alfsen and Shultz ${ }^{22,23}$ and a characterization result concerning the low rank homogeneous spaces of Ito and Lourenço. ${ }^{24}$ At this point, sequential product spaces have been established to be EJAs but only in a rather indirect way. In Sec. IV, we directly construct the Jordan product using the sequential product. In Sec. V, we show how the additional requirement of local tomography forces the sequential product space to be a $\mathrm{C}^{*}$-algebra. Section VI establishes an infinite-dimensional generalization of the main theorem, while in Sec. VII we discuss how changing the axioms of a sequential product impacts the results of this paper.

\section{PRELIMINARIES}

As mentioned in the Introduction, our main goal is to show that a sequential product space is homogeneous and self-dual, so let us start therefore with the definition of these properties.

Definition 5. Let $V$ be an order unit space. An order isomorphism is an invertible linear map $\Phi: V \rightarrow V$ such that $\Phi(a) \geq 0 \Leftrightarrow a \geq 0$ for all $a \in V$. Denote the interior of the positive cone of $V$ by $C$, i.e., $a \in C \Longleftrightarrow \exists \epsilon \in \mathbb{R}_{>0}: \epsilon 1 \leq a$. We call $V$ homogeneous when for all $a, b \in C$ there exists an order isomorphism $\Phi$ such that $\Phi(a)=b$.

Definition 6. Let $V$ be an order unit space. We call $V$ self-dual when there exists an inner product $\langle\cdot, \cdot\rangle$ such that for all $a \in V: a \geq 0$ if and only if $\langle a, b\rangle \geq 0$ for all $b \geq 0$.

To give a complete picture of the theory of sequential product spaces, we will repeat some of the known basic results regarding sequential products and sequential product spaces that can be found in, for instance, Refs. 11, 16, and 25. This section on preliminaries will end with the existence proofs of spectral decompositions of effects and the corollary of homogeneity that follows from it, which was originally shown in Ref. 16 (in a slightly different setting).

Unless otherwise stated, we will let $V$ denote a finite-dimensional sequential product space, $E=[0,1]_{V}$ denote its set of effects, and $\&: E \times E \rightarrow E$ denote a sequential product. For $a \in E$, we let $a^{\perp}=1-a$ denote its complement which by virtue of $a$ lying in the unit interval of $V$ is also an effect.

Proposition 1. Let $a, b, c \in E$.

1. $a \& 0=0 \& a=0$ and $a \& 1=1 \& a=a$.

2. $a \& b \leq a$.

3. If $a \leq b$, then $c \& a \leq c \& b$.

Proof. Originally proved in Ref. 11.

1. We of course have $a \mid a$, and by S6, we have $a \mid a^{\perp}$. Using S6 again, we then see that $a \mid a+a^{\perp}=1$ so that by S3 $1 \& a=a \& 1=a$. Using S6 again, we also have $a \mid 1^{\perp}=0$ so that it remains to show that $a \& 0=0$. By S1, we get $a \& 0=a \&(0+0)=a \& 0+a \& 0$ so that indeed $a \& 0=0$.

2. By the previous point and S1, $a=a \& 1=a \&(b+(1-b))=a \& b+a \&(1-b)$ so that indeed $a \& b \leq a$, as $a \&(1-b) \geq 0$.

3. Since $a \leq b$, we have $b-a \geq 0$ so that using S1 we have $c \& b=c \&(b-a+a)=c \&(b-a)+c \& a$, from which we derive $c$ \& $(b-a)$ $=c \& b-c \& a$. Since the left-hand side is greater than zero, the right-hand side must be as well. 
Proposition 2. Let $a, b \in E$, and let $q$ be any rational number between zero and one, and $\lambda$ be any real number between zero and one.

1. $a \&(q b)=q(a \& b)$.

2. $\quad a \&(\lambda b)=\lambda(a \& b)$.

3. $(\lambda a) \& b=a \&(\lambda b)=\lambda(a \& b)$.

4. If $a \mid b$, then $a \mid \lambda b$.

Proof. Originally proved in Ref. 16.

1. Of course $a \& b=a \&\left(n \frac{1}{n} b\right)=n a \&\left(\frac{1}{n} b\right)$ by S1. Dividing by $n$ gives $a \&\left(\frac{1}{n} b\right)=\frac{1}{n}(a \& b)$. By summing this equation multiple times, we see that we get $a \&(q b)=q(a \& b)$ for any rational $0 \leq q \leq 1$.

2. Let $q_{i}$ be an increasing sequence of positive rational numbers that converges to $\lambda$. Using the norm of the order unit space, we compute

$$
\begin{aligned}
\|\lambda(a \& b)-a \&(\lambda b)\| & =\left\|\left(\lambda-q_{i}\right)(a \& b)+q_{i}(a \& b)-a \&(\lambda b)\right\| \\
& =\left\|\left(\lambda-q_{i}\right)(a \& b)-a \&\left(\left(\lambda-q_{i}\right) b\right)\right\| .
\end{aligned}
$$

Because $\left(\lambda-q_{i}\right) b \leq\left(\lambda-q_{i}\right)\|b\| 1$ and using Proposition 1.3, we have $\left\|a \&\left(\left(\lambda-q_{i}\right) b\right)\right\| \leq\|a\|\left\|\left(\lambda-q_{i}\right) b\right\|=\left(\lambda-q_{i}\right)\|a\|\|b\|$. Then, $\|\lambda(a \& b)-a \&(\lambda b)\| \leq 2\left(\lambda-q_{i}\right)\|a\|\|b\|$. This expression indeed goes to zero as $i$ increases so that indeed $\lambda(a \& b)=a \&(\lambda b)$.

3. Clearly, $\frac{1}{n} a \mid \frac{1}{n} a$ so that by S6 $\frac{1}{n} a \mid a$. In the same way, we also get $q a \mid a$ and $q a^{\perp} \mid a^{\perp}$ for any rational $0 \leq q \leq 1$. Using the rule $a|b \Rightarrow a| b^{\perp}$ from S6, we then also get $q a^{\perp} \mid a$ so that $a \mid\left(q a+q a^{\perp}\right)=q 1$. Then, indeed $(q 1) \& a=a \&(q 1)=q(a \& 1)=q a$ so that also $(q a) \& b$ $=(a \&(q 1)) \& b=a \&((q 1) \& b))=a \& q b=q(a \& b)$. Now, let $\lambda \in[0,1]$ be a real number and let $q_{i}$ be a sequence of rational numbers converging to $\lambda$ so that also $q_{i} a \rightarrow \lambda a$ and $q_{i}(a \& b) \rightarrow \lambda(a \& b)$. Then, $q_{i}(a \& b)=\left(q_{i} a\right) \& b \rightarrow(\lambda a) \& b$ by S2. We conclude that $(\lambda a) \& b=\lambda(a \& b)=a \&(\lambda b)$.

4. Suppose $a \mid b$. Using the previous point, $a \&(\lambda b)=\lambda(a \& b)=\lambda(b \& a)=(\lambda b) \& a$.

As a result of this proposition, the left-multiplication map $L_{a}: E \rightarrow E$ for $a \in E$ given by $L_{a}(b)=a$ \& $b$ can be extended by linearity to the entirety of $V$ by $L_{a}(b-c)=L_{a}(b)-L_{a}(c)$. Similarly, we can define the sequential product for any element in the positive cone of $V$ (not necessarily below the identity) by rescaling: $a \& b:=\|a\|\left(\left(\frac{1}{\|a\|} a\right) \& b\right)$.

Definition 7. An effect $p \in E$ is called sharp when the only effect below both $p$ and $p^{\perp}$ is the zero effect, i.e., when the following implication holds: $b \leq p$ and $b \leq p^{\perp}$ implies $b=0$.

When $V=B(H)^{\text {sa }}$, the sharp effects are precisely the projections. This should be clear considering the following proposition:

Proposition 3. Let $a \in E$ be an effect. It is $a$ is sharp if and only if $a \& a^{\perp}=0$ if and only if $a \& a=a$.

Proof. Originally proved in Ref. 11.

The equivalence of $a \& a^{\perp}=0$ and $a \& a=a$ follows straightforwardly by S1 and Proposition 1.1: $a=a \& 1=a \&\left(a+a^{\perp}\right)$ $=a \& a+a \& a^{\perp}$.

So let us assume $a$ is sharp. By S6, we have $a \mid a^{\perp}$ so that $a \& a^{\perp}=a^{\perp} \& a$. By 1.2, we have $a \& a^{\perp} \leq a$ and similarly $a^{\perp} \& a \leq a^{\perp}$, and the expression $a \& a^{\perp}$ is therefore below both $a$ and $a^{\perp}$ and by sharpness of $a$ has to be zero. Now, suppose $a \& a^{\perp}=0$ and let $b \leq a$ and $b \leq a^{\perp}$. If $b \leq a^{\perp}$, then by 1.2 we get $a \& b \leq a \& a^{\perp}=0$, and similarly, we get $a^{\perp} \& b=0$. By S4, we have $b \mid a$ and $b \mid a^{\perp}$ so that $b=b \& 1=b \&\left(a+a^{\perp}\right)=$ $b \& a+b \& a^{\perp}=a \& b+a^{\perp} \& b=0+0=0$.

Let us now introduce the notion of orthogonal effects which was hinted at in S4:

Definition 8. We call two effects $p$ and $q$ orthogonal when $p \& q=0$.

Of course, by $\$ 4$, orthogonality is a symmetric relation, and we note that therefore orthogonal effects are also compatible.

Definition 9. Let $a \in E$ be an effect. We define the powers of $a$ inductively to be $a^{0}:=1$ and $a^{n}:=a$ \& $a^{n-1}$. We define the classical algebra of $a$ to be the linear space $C(a)$ spanned by all the powers of $a$ and $a^{\perp}$.

Proposition 4. Let $a \in E$ be an effect. $C(a)$ is a commutative sequential product space. 
Proof. $C(a)$ inherits the order structure from $V$ in the obvious way. Of course, $a \mid a^{\perp}$, and because of S7, we have $a^{n} \mid\left(a^{\perp}\right)^{m}$ for all $n$ and $m$. Because of S6 and Proposition 2.4, linear combinations of compatible effects are also compatible and hence all effects of $C(a)$ are compatible.

We are now in a position to use the seminal representation theorem from the work of Kadison:

Proposition 5 (Ref. 26). Let $V$ be a complete order unit space with a bilinear operation $\circ$ that preserves positivity: $a \circ b \geq 0$ when $a, b \geq 0$. Then, there exists a compact Hausdorff space $X$ such that $V \cong C(X)$, the space of continuous real-valued functions on $X$. This isomorphism is both an order- and algebra-isomorphism.

Proposition 6. Let $a \in E$ be an effect. $C(a) \cong \mathbb{R}^{n}$ for some $n \in \mathbb{N}$.

Proof. The sequential product is linear in the second argument. Since $C(a)$ is a commutative sequential product space by Proposition 4 , its product is also linear in the first argument, and hence, this operation is bilinear. It obviously preserves positivity by definition so that Kadison's theorem applies and $C(a) \cong C(X)$. Since $V$ is finite-dimensional, $C(a)$ has to be so as well, and hence, $C(X)$ is finite-dimensional. Of course, $C(X)$ is finite-dimensional only when $X$ is finite so that $X$ is necessarily discrete. We conclude that indeed $C(X) \cong \mathbb{R}^{n}$.

Corollary 7. Let $a \in E$ be an effect. There exists a set of orthogonal sharp effects $p_{i}$ compatible with $a$ and positive scalars $\lambda_{i}$ such that $a=\sum_{i} \lambda_{i} p_{i}$

Proof. By the previous proposition, $C(a) \cong \mathbb{R}^{n}$, and this space is obviously spanned by orthogonal sharp effects; hence, we can find the desired $p_{i}$ and $\lambda_{i}$. By construction, $p_{i} \in C(a)$ so that they are compatible with $a$.

We will refer to a decomposition of $a$ in the above sense as a spectral decomposition of $a$. The existence of this decomposition is already enough to show that the space must be homogeneous:

Proposition 8. Let $C$ denote the cone of strictly positive elements in $V$, i.e., the elements $v \in V$ such that $\exists \epsilon>0$ with $\epsilon 1 \leq v$. The cone $C$ is homogeneous, i.e., for every $v, w \in C$, there exists an order isomorphism $\Phi: V \rightarrow V$ such that $\Phi(v)=w$.

Proof. Originally proved in Ref. 16.

For an arbitrary positive element $a$, we can find a spectral decomposition $a=\sum_{i} \lambda_{i} p_{i}$ such that $\lambda_{i}>0$, i.e., we do not write the zero "eigenvalues." It is then straightforward to check that $a$ lies in the interior of the positive cone if and only if $\sum_{i} p_{i}=1$. In that case, we define its inverse $a^{-1}=\sum_{i} \lambda_{i}^{-1} p_{i}$. Since the $p_{i}$ are all compatible and $a$ and $a^{-1}$ are linear combinations of these effects, they are also compatible and we calculate $a \& a^{-1}=\sum_{i, j} \lambda_{i} \lambda_{j}^{-1} p_{i} \& p_{j}=\sum_{i} \lambda_{i} \lambda_{i}^{-1} p_{i}=\sum_{i} p_{i}=1$ so that $a^{-1}$ is indeed the inverse of $a$ with respect to the sequential product. The multiplication map $L_{a}(b):=a \& b$ is positive and has a positive inverse $L_{a^{-1}}$ due to S5: $a^{-1} \&(a \& b)=\left(a^{-1} \& a\right) \& b=1 \& b=b$. The map $L_{a}$ is therefore an order isomorphism when $a$ is strictly positive. Now, for $a$ and $b$ strictly positive and hence invertible, define $\Phi: V \rightarrow V$ by $\Phi=L_{b} L_{a^{-1}}$. As this is a composition of order isomorphisms, it is also an order isomorphism and of course $\Phi(a)=b \&\left(a^{-1} \& a\right)=b \& 1=b$ as desired.

\section{PROOF OF SELF-DUALITY}

With homogeneity of $V$ now established, we set our sights on proving self-duality. We do this in a few steps. First, we study the lattice of sharp effects in Sec. III A. We then consider properties of the atoms of this lattice in Sec. III B. Then, in Sec. III C, we establish that this lattice has the covering property as defined in Ref. 22. The covering property states that for every sharp effect $p$ there is a unique number $r$ called the rank of $p$ such that we can write $p=\sum_{i=1}^{r} p_{i}$, where $p_{i}$ are atomic and orthogonal. Using this definition, we can define the rank of a space as equal to the rank of the unit effect. The existence of the well-defined ranks of sharp effects allows us to reduce the question of self-duality to that of self-duality in rank 2 spaces. This problem is in turn solved by appealing to the classification result of Ref. 24 that homogeneous spaces of rank 2 are always self-dual, which is done in Sec. III D.

\section{A. The lattice of sharp effects}

Proposition 9. Let $a \in E$ be any effect, and let $p \in E$ be sharp.

1. $a \leq p$ if and only if $p \& a=a \& p=a$ if and only if $p^{\perp} \& a=0$.

2. $p \leq a$ if and only if $p \& a=a \& p=p$.

Proof.

1. Suppose $a \leq p$ with $p$ sharp. Then, $p^{\perp} \& a \leq p^{\perp} \& p=0$ by Propositions 3 and 1.2. Hence, $a \mid p^{\perp}$ and $a \mid p$ so that $a=a \&\left(p+p^{\perp}\right)=a \& p$ $+a \& p^{\perp}=a \& p=p \& a$. For the other direction, we note that $a=p \& a \leq p$ by 1.2 proposition. 
2. Suppose $p \leq a$ with $p$ sharp; then $a^{\perp} \leq p^{\perp}$ with $p^{\perp}$ sharp so that by the previous point $a \mid p$ and $p \& a^{\perp}=0$ so that $p=p \&\left(a+a^{\perp}\right)=p \&$ a.

Definition 10. For an effect $a \in E$, we let $\lceil a\rceil$ denote the smallest sharp element above $a$ (when it exists) and $\lfloor a\rfloor$ denote the largest sharp element below $a$ (when it exists).

Proposition 10. The ceiling and the floor exist for any $a$. Moreover, writing $a=\sum_{i} \lambda_{i} p_{i}$ with $1 \geq \lambda_{i}>0$ and the $p_{i}$ sharp and orthogonal, then $\lceil a\rceil=\sum_{i} p_{i}$ and $\lfloor a\rfloor=\left\lceil a^{\perp}\right\rceil^{\perp}$.

Proof. Write $a=\sum_{i} \lambda_{i} p_{i}$. Of course, $\sum_{i} p_{i}$ is an upper bound of $a$. Suppose $a \leq r$ for some sharp $r$. Then, $\lambda_{i} p_{i} \leq r$, so by Proposition $9.1 r \&$ $\left(\lambda_{i} p_{i}\right)=\lambda_{i} p_{i}$. Using linearity, we can rewrite this expression to $\lambda_{i}\left(r \& p_{i}\right)=\lambda_{i} p_{i}$ so that $r \& p_{i}=p_{i}$. So, again by $9.1, p_{i} \leq r$ and $p_{i} \mid r$ from which we get $r \& \sum_{i} p_{i}=\sum_{i} r \& p_{i}=\sum_{i} p_{i}$ so that also $\sum_{i} p_{i} \leq r$ which proves that it is the least upper bound. The other statement now follows because $a \leq b \Leftrightarrow b^{\perp} \leq a^{\perp}$.

As a corollary of the above, we also see that $\lceil\lambda a\rceil=\lceil a\rceil$ when $1 \geq \lambda>0$ and that $a$ is sharp if and only if $\lceil a\rceil=a$ or $\lfloor a\rfloor=a$. We also note that $a \leq b$ implies that $\lceil a\rceil \leq\lceil b\rceil$.

Proposition 11. The sharp effects form a lattice: for two sharp effects $q$ and $p$, their least upper bound $p \vee q$ and greatest lower bound $p \wedge q$ exist. Furthermore, the following relation holds between them: $(p \vee q)^{\perp}=p^{\perp} \wedge q^{\perp}$.

Proof. We claim that $p \vee q=\left\lceil\frac{1}{2}(p+q)\right\rceil$. Note that $p \leq p+q$ and thus that $\frac{1}{2} p \leq \frac{1}{2}(p+q)$ so that $p=\lceil p\rceil=\left\lceil\frac{1}{2} p\right\rceil \leq\left\lceil\frac{1}{2}(p+q)\right\rceil$. Similarly, we also have $q \leq\left\lceil\frac{1}{2}(p+q)\right\rceil$, and thus, this is an upper bound. Suppose now that $p \leq a$ and $q \leq a$ for some sharp $a$. Then, of course also $\frac{1}{2}(p+q) \leq \frac{1}{2}(a+a)=a$. Taking the ceiling on both sides then shows that indeed $p \vee q=\left\lceil\frac{1}{2}(p+q)\right\rceil$.

To find $p \wedge q$, we note that $(\cdot)^{\perp}$ is an order-antiautomorphism and thus that it interchanges joins with meets: $(p \vee q)^{\perp}=p^{\perp} \wedge q^{\perp}$.

Proposition 12. Let $a \in E$ be any effect, and let $p \in E$ be sharp.

1. $p \& a=0$ if and only if $p+a \leq 1$ in which case it is the least upper bound of the two. When $p \& a=0$, their sum $p+a$ is sharp if and only if $a$ is also sharp.

2. If both $a$ and $p$ are sharp and $a$ and $p$ are compatible, then $p \& a$ is sharp and equal to their join: $p \wedge a=p \& a$.

Proof. Originally proved in Ref. 11 .

1. $p \& a=0$ if and only if $p^{\perp} \& a=a$ which by Proposition 9.2 is true if and only if $a \leq p^{\perp}=1-p$ so that indeed $p+a \leq 1$. That $p+a$ is an upper bound of $p$ and $a$ is obvious. Suppose now that $b$ is also an upper bound so that $p \leq b$ and $a \leq b$. We then calculate using Proposition $9.2 p=p \& b=p \&(b-a+a)=p \&(b-a)+p \& a=p \&(b-a)$ so that $p \leq b-a$ again by 9.2. This gives $p+a \leq b$ so that $p+a$ is indeed the least upper bound.

Now, to show $p+a$ is sharp if and only if both $p$ and $a$ are sharp, since $p \& a=0$, we have $p \mid a$ and thus also $p \mid p+a$ and $a \mid p+a$ by S6. We calculate $(p+a) \&(p+a)=p \& p+2 p \& a+a \& a=p+a \& a=(p+a)-(a-a \& a)$. We therefore have $(p+a) \&(p+a)=p+a$ if and only if $a-a \& a=0$, which proves the result by Proposition 3 .

2. Suppose both $p$ and $a$ are sharp and that $p \mid a$. We calculate

$$
(p \& a) \&(p \& a)=(p \& a) \&(a \& p)=p \&(a \&(a \& p))=p \&(a \& p)=p \&(p \& a)=p \& a,
$$

where we have used that $a \mid a \& p$ and $p \mid a \& p$ by S7. Hence, $p \& a$ is sharp. It is a lower bound of $p$ and $a$ by 1.2. Suppose $b \leq p, a$ is also a lower bound. We calculate $p \& a=p \&(a-b+b)=p \&(a-b)+p \& b=p \&(a-b)+b \geq b$, where we have used that $p \& b=b$ as a consequence of Proposition 9.1.

Lemma 13. Let $a, b \in E$. If $b \& a=0$, then $b \&\lceil a\rceil=0$.

Proof. Write $a=\sum_{i} \lambda_{i} p_{i}$. If $b \& a=0=\sum_{i} \lambda_{i} b \& p_{i}$, then we must have $b \& p_{i}=0$ for all $p_{i}$. Since $\lceil a\rceil=\sum_{i} p_{i}$, the claim follows.

Lemma 14. Let $p \in E$ be sharp and $a \in E$ arbitrary, then $\lceil p \& a\rceil=\lceil p \&\lceil a\rceil\rceil$.

Proof. First of all, $p \& a \leq p \&\lceil a\rceil$ so that $[p \& a\rceil \leq\lceil p \&\lceil a\rceil\rceil$. It suffices therefore to prove the other inequality. Because $p \& a \leq p$ $\& 1=p$, we also have $\lceil p \& a\rceil \leq\lceil p\rceil=p$, implying $\lceil p \& a\rceil^{\perp} \& p=0$ so that $\lceil p \& a\rceil^{\perp}$ and $p$ are compatible. Now, because $p \& a \leq\lceil p \& a\rceil$, we can use Proposition 9.1 to write $0=\lceil p \& a\rceil^{\perp} \&(p \& a)=\left(\lceil p \& a\rceil^{\perp} \& p\right) \& a=\left(\lceil p \& a\rceil^{\perp} \& p\right) \&\lceil a\rceil=\lceil p \& a\rceil^{\perp} \&(p \&\lceil a\rceil)$ where we have used Lemma 13 to replace $a$ with $\lceil a\rceil$. Since $\lceil p \& a\rceil^{\perp} \&(p \&\lceil a\rceil)=0$, we use 9.1 again to conclude $p \&\lceil a\rceil \leq\lceil p \& a\rceil$ so that indeed $\lceil p \&\lceil a\rceil\rceil \leq\lceil p \& a\rceil$. 


\section{B. Atomic effects}

Definition 11. A nonzero sharp effect $p \in E$ is atomic if for all $a \in E$ with $a \leq p$ we have $a=\lambda p$ for some $\lambda \in[0,1]$.

The name atomic comes from the fact that in the lattice of sharp effects, the atomic effects are the smallest nonzero elements. It turns out that the lattice of effects in a finite-dimensional sequential product space is atomic, meaning that any sharp effect can be written as a sum of atomic effects:

Proposition 15. Every sharp effect can be written as a sum of orthogonal atomic effects.

Proof. Let $p$ be sharp and suppose it is not atomic. We can find $0 \leq a \leq p$ such that $a \neq \lambda p$ for any $\lambda \in[0,1]$. Write $a=\sum_{i} \lambda_{i} q_{i}$, where the $q_{i}$ are sharp and orthogonal. Then, $\lambda_{i} q_{i} \leq p$ and thus also $\left\lceil\lambda_{i} q_{i}\right\rceil=q_{i} \leq\lceil p\rceil=p$. If all $q_{i}$ are equal to $p$, then $a$ is a multiple of $p$, so at least one of the $q_{i}$ is strictly smaller than $p$. We can repeat this process for $q_{i}$ and $p-q_{i}$, getting a sequence of nonzero orthogonal sharp effects that sum up to $p$. Since the space is finite-dimensional and orthogonal effects are linearly independent, this process must stop after a finite amount of steps in which case we are left with atomic effects.

Corollary 16. Every $a \in V$ can be written as $a=\sum_{i} \lambda_{i} p_{i}$, where $p_{i}$ are orthogonal sharp atomic effects.

Proof. For every $a \in V$, we can find a spectral decomposition in terms of orthogonal sharp effects. The previous proposition shows that these sharp effects can be further decomposed into atomic effects.

Recall that the norm in an order unit space is defined as $\|a\|:=\inf \{r ;-r 1 \leq a \leq r 1\}$.

Lemma 17. A nonzero effect $p$ is atomic if and only if we have $p \& a=\|p \& a\| p$ for all $a \in E$.

Proof. First, we establish that the norm of any nonzero sharp effect is equal to 1 . Let $q$ be sharp. We see that $q=q \& q \leq q \&(\|q\| 1)$ $=\|q\| q \& 1=\|q\| q$ so that $\|q\| \geq 1$. But since $q \leq 1$, we also have $\|q\| \leq 1$.

Suppose $p$ is atomic. Because $0 \leq p \& a \leq p$, we must have $p \& a=\lambda p$ for some $0 \leq \lambda \leq 1$ so that $\|p \& a\|=\lambda\|p\|=\lambda$ because $p$ is sharp.

For the other direction, first note that $p=p \&[p]=\|p \&[p\rceil\| p=\|p\| p$ so that necessarily $\|p\|=1$ (since $p \neq 0$ ). By writing $p$ as a linear combination of sharp effects, we see that then also $\left\|p^{2}\right\|=1$. Now, $p \& p=\left\|p^{2}\right\| p=p$ so that $p$ is sharp. Let $q \leq p$ be nonzero sharp. Then, $q$ $=p \& q=\|p \& q\| p=p$ (using again that $\|q\|=1$ because $q$ is sharp), so there are no nontrivial sharp effects below $p$. Now, if $a=\sum{ }_{i} \lambda_{i} q_{i}$ lies below $p$, we see that $\lambda_{i} q_{i} \leq p$ so that $\left[\lambda_{i} q_{i}\right\rceil=q_{i} \leq\lceil p\rceil=p$ so that $q_{i}=p$ and thus $a=\lambda p$. Since all $a \in E$ can be written in this way, we conclude that this holds for all $a \leq p$ so that $p$ is indeed atomic.

Corollary 18. The set of atomic effects is closed in the norm topology.

Proof. Let $p_{n} \rightarrow p$ be a norm converging set of atomic effects $p_{n}$. We need to show that $p$ is also atomic. As a result of the previous lemma, we have $p_{n} \& a=\left\|p_{n} \& a\right\| p_{n}$ for all effects $a$. By continuity of \& (i.e., axiom S2), we have $p_{n} \& a \rightarrow p \& a$ so that $p \& a=\lim p_{n} \& a$ $=\lim \left\|p_{n} \& a\right\| p_{n}=\|p \& a\| p$. Using the previous lemma again, we conclude that $p$ is indeed atomic.

Proposition 19. Let $a \in E$ be arbitrary and $p \in E$ be atomic, then $a \& p$ is proportional to an atomic effect.

Proof. The property that $0 \leq a \leq p \Rightarrow a=\lambda p$ is determined by the order, so any order isomorphism preserves it. If $a$ is invertible, then $L_{a}: V \rightarrow V$ given by $L_{a}(b):=a \& b$ is an order isomorphism so that $L_{a}(p)$ must be proportional to an atomic effect. For noninvertible $a$, we write $a_{n}=a+\frac{1}{n}$ so that $a_{n}$ is invertible and the sequence $a_{n}$ converges to $a$. Let $q_{n}=\left(a_{n} \& p\right) /\left\|a_{n} \& p\right\|$. We see that all all $q_{n}$ are atomic. By the continuity condition S2, we get $a_{n} \& p \rightarrow a \& p$ so that also $\left\|a_{n} \& p\right\| \rightarrow\|a \& p\|$. The sequence $q_{n}$ is therefore also convergent, and since the set of atomic effects is closed by the previous corollary, we conclude that $q_{n} \rightarrow q=(a \& p) /\|a \& p\|$ is atomic.

\section{The covering property}

At this point, we know that the set of sharp effects forms an atomic lattice, but in fact it has the much stronger covering property that allows us to attach a rank to each sharp effect: the amount of atomic effects needed to make the effect. To show this, we need some results from the work of Alfsen and Shultz ${ }^{23}$ that unfortunately were proven in a slightly different setting. We will repeat these results with very similar proofs but adapted to work in the setting of sequential product spaces. 
Lemma 20 (Lemma 8.9 of Ref. 23): Let $q$ and $p$ be sharp with $q \leq p$, then $p-q=p \wedge q^{\perp}$.

Proof. Let $q$ and $p$ be sharp with $q \leq p$, then $p-q$ is sharp and $p \mid q$, so also $p \mid q^{\perp}$ so that $p-q=p \& q^{\perp}=p \wedge q^{\perp}$ by Proposition 12.2 .

Lemma 21 (Ref. 23). (Theorem 8.32): Let $p$ be sharp and $a$ arbitrary, then $\lceil p \& a\rceil=\left(\lceil a\rceil \vee p^{\perp}\right) \wedge p$.

Proof. Because $\lceil p \& a\rceil=\lceil p \&\lceil a\rceil\rceil$, by Lemma 14, it suffices to prove this for sharp $a$. We prove the equality by showing that an inequality holds in both directions.

Since $p^{\perp} \leq a \vee p^{\perp}$, we have $p^{\perp} \mid\left(a \vee p^{\perp}\right)$ by Proposition 9.1 so that in turn $p \mid\left(a \vee p^{\perp}\right)$ by S6. We proceed by using S5: $\left(a \vee p^{\perp}\right) \&(p \& a)$ $=\left(\left(a \vee p^{\perp}\right) \& p\right) \& a=p \&\left(\left(a \vee p^{\perp}\right) \& a\right)=p \& a$ because $a \vee p^{\perp} \geq a$. Therefore, $p \& a \leq a \vee p^{\perp}$ which implies that $[p \& a] \leq a \vee p^{\perp}$. Since also $p \& a \leq p$ and therefore $\lceil p \& a\rceil \leq p$, we conclude that $\lceil p \& a\rceil \leq\left(a \vee p^{\perp}\right) \wedge p=\left(\lceil a\rceil \vee p^{\perp}\right) \wedge p$.

Now, for the other direction, we obviously have $p^{\perp} \&(p \& a)=\left(p^{\perp} \& p\right) \& a=0$ by S6 and S5 so that by Lemma $13 p^{\perp} \&\lceil p \& a\rceil=0$. Using Proposition 9.1, we see then that $p \mid\lceil p \& a\rceil^{\perp}$ and therefore by Proposition 12.2 that $\lceil p \& a\rceil^{\perp} \& p=\lceil p \& a\rceil^{\perp} \wedge p$. Since $p \& a \leq\lceil p \& a\rceil$, we calculate using Proposition 9.1: $0=\lceil p \& a\rceil^{\perp} \&(p \& a)=\left(\lceil p \& a\rceil^{\perp} \& p\right) \& a=\left(\lceil p \& a\rceil^{\perp} \wedge p\right) \& a$ so that $a \leq\left(\lceil p \& a\rceil^{\perp} \wedge p\right)^{\perp}=\lceil p \& a\rceil \vee p^{\perp}$ by Proposition 11. Then, of course also $a \vee p^{\perp} \leq[p \& a\rceil \vee p^{\perp}$, and by noting that $[p \& a\rceil$ and $p^{\perp}$ are orthogonal and using Proposition 12.1, $\lceil p \& a\rceil \vee p^{\perp}=\lceil p \& a\rceil+p^{\perp}$. Bringing $p^{\perp}$ to the other side and using Lemma 20 (which applies because $\left.p^{\perp} \leq a \vee p^{\perp}\right)$ then gives $\left(a \vee p^{\perp}\right) \wedge p=$ $a \vee p^{\perp}-p^{\perp} \leq\lceil p \& a\rceil$.

Proposition 22 (Ref. 23). (Proposition 9.7): The lattice of sharp effects has the covering property: for $q$ atomic, the expression $(q \vee p) \wedge$ $p^{\perp}=(q \vee p)-p$ is either zero or atomic. In other words, when $q$ does not lie below $p$, then there is no sharp effect lying strictly between $p$ and $q \vee p$.

Proof. By the previous lemma, $(q \vee p) \wedge p^{\perp}=\left\lceil p^{\perp} \& q\right\rceil$. Since $p^{\perp} \& q$ is proportional to an atom by Proposition 19, it is either zero (when $q \leq p)$ in which case we are done or nonzero in which case $\left[p^{\perp} \& u\right\rceil$ is an atom, which also proves the statement. The equality $(q \vee p) \wedge p^{\perp}$ $=(q \vee p)-p$ follows directly from Lemma 20 .

The last observation is proven as follows. Suppose $p<r<q \vee p$. Subtract $p$ to get $0<r-p<q \vee p-p$. As $r-p$ is sharp and $q \vee p-p$ has been established to be atomic, this is not possible.

Definition 12. Let $p$ be sharp, and let $p_{i}$ be a collection of atomic orthogonal effects such that $p=\sum_{i}^{n} p_{i}$. The minimal size of such a collection is called the rank of $p$. The rank of a sequential product space is defined to be the rank of the unit effect.

With the covering property proven, we can finally prove the following "dimension" theorem:

Proposition 23 (Ref. 22). (Proposition 1.66) Write $p=\sum_{i}^{n} p_{i}$, where the $p_{i}$ are orthogonal and atomic, then $n=\operatorname{rnk} p$, i.e., all ways of writing $p$ as a sum of atomic effects require an equal amount of atomic effects. Furthermore, suppose $q \leq p$, then rnk $q \leq \operatorname{rnk} p$, and if also rnk $q=\operatorname{rnk} p$, then necessarily $q=p$.

Proof. Let $p^{\prime}=p_{1} \vee \ldots \vee p_{n-1}$. Then, $p^{\prime} \vee p_{n}=p$, and by the covering property (Proposition 22), there is no sharp effect strictly between $p^{\prime}$ and $p$. Suppose now $q \leq p$ is atomic, and suppose that $q$ is not below $p^{\prime}$. Then, $p^{\prime} \vee q$ must be strictly greater than $p^{\prime}$, but since this must also lie below $p$, we conclude that $p^{\prime} \vee q=p$.

Let $p=\sum_{j}^{r} q_{j}=q_{1} \vee \cdots \vee q_{r}$, where $r:=\operatorname{rnk} p$ is the minimal amount of terms needed to write $p$ as a sum of atomic effects. We must then of course have $r \leq n$. Let $q=q_{2} \vee \ldots \vee q_{r}$, then $q$ must lie strictly below $p$ since $q_{1} \leq p$ but not $q_{1} \leq q$. It then follows that there must be a $p_{i}$ such that $p_{i}$ does not lie below $q$ as well since otherwise $p=p_{1} \vee \ldots \vee p_{n} \leq q<p$. Without loss of generality, let this $p_{i}$ be $p_{1}$. By the previous paragraph, we must have $p_{1} \vee q=p_{1} \vee q_{2} \ldots \vee q_{r}=p$. This procedure can be repeated with $q_{2}, \ldots, q_{r}$ until we are left with the equation $p_{1} \vee \ldots \vee p_{r}=p$. Suppose $n>r$, then $p_{n}$ is orthogonal to all the other $p_{i}$ 's, we have, in particular, $p_{n} \leq p_{1}^{\perp} \wedge \cdots \wedge p_{r}^{\perp}=\left(p_{1} \vee \cdots \vee p_{r}\right)^{\perp}$ $=p^{\perp}$. Since also $p_{n} \leq p$, we get $p_{n}=0$ by sharpness which is a contradiction. We therefore have $n=r$.

Now, suppose $q=\sum_{j}^{s} q_{j} \leq p=\sum_{i}^{r} p_{i}$, where $s=\operatorname{rnk} q$. Since $p-q$ is sharp, we can write $p-q=\sum_{k}^{t} v_{k}$. Then, because $p=\sum_{j}^{s} q_{j}+\sum_{k}^{t} v_{k}$, we must by the previous points have $s+t=r$ so that indeed $\operatorname{rnk} q \leq \operatorname{rnk} p$. When these ranks are equal, we must have $t=0$ so that indeed $p-q=0$.

Corollary 24. Let $p \neq q$ be two atomic sharp effects and suppose $0 \leq a \leq p \vee q$, then $a=\lambda_{1} r_{1}+\lambda_{2} r_{2}$, where $r_{i}$ are orthogonal and atomic and $r_{1}+r_{2}=p \vee q$.

Proof. By Proposition 22, $(p \vee q)-p$ is atomic so that $p \vee q$ can be written as the sum of two atomic sharp effects so that indeed rnk $p \vee$ $q=2$. Suppose $0 \leq a \leq p \vee q$. Let $a=\sum_{i}^{n} \lambda_{i} r_{i}$ be a spectral decomposition of $a$ with $r_{i}$ orthogonal and atomic. Of course, $\lceil a\rceil \leq p \vee q$ so that by the previous proposition we must have $\operatorname{rnk}\lceil a\rceil \leq 2$. Since also by the previous proposition $\operatorname{rnk} \sum_{i}^{n} r_{i}=n$, we see that we must have $n=2$ and thus that $a$ is as desired. 


\section{Self-duality}

The important concept of this section will be that of strict convexity of a cone since this is related to a characterization theorem for homogeneous spaces.

Definition 13. Let $C$ be a positive cone of an order unit space $V$. We call $F \subseteq C$ a face of $C$ if $F$ is a convex set such that whenever $\lambda a+(1-\lambda) b \in F$ with $0<\lambda<1$, then $a, b \in F$. The face $\left\{\lambda p ; \lambda \in \mathbb{R}_{\geq 0}\right\}$ of $C$ defined by an extreme point $p \in C$ is called an extreme ray. A face is called proper when it is nonempty and not equal to $C$. If the only proper faces of a cone are extreme rays, the cone is strictly convex.

Proposition 25 (Ref. 24). Let $V$ be a finite-dimensional ordered vector space with a strictly convex homogeneous positive cone, then $V$ is order isomorphic to a spin-factor, i.e., $V \cong H \oplus \mathbb{R}$, where $H$ is a real finite-dimensional Hilbert space with the order on $H \oplus \mathbb{R}$ given by $(v, t)$ $\geq 0 \Leftrightarrow t \geq\|v\|_{2}$.

Definition 14. Let $p$ and $q$ be two unequal atomic effects. We define the order ideal generated by $p$ and $q$ as $V_{p, q}:=\{v \in V ; \exists n:-n p \vee q \leq$ $v \leq n p \vee q\}$.

$V_{p, q}$ is an order unit space with unit $p \vee q$. If we have $a, b \in[0,1]_{p, q}$, then $a \& b \leq a \leq p \vee q$ so that the sequential product restricts to this space. We therefore conclude by Proposition 8 that this space has a homogeneous positive cone.

Lemma 26. Let $p$ and $q$ be two unequal atomic effects. The positive cone of $V_{p, q}$ is strictly convex.

Proof. Let $F$ be a proper face of the positive cone of $V_{p, q}$. Let $a \in F$ and write $a=\lambda\left(\lambda^{-1} a\right)+\lambda^{\perp} 0$ so that $\lambda^{-1} a \in F$. We see that $F$ is closed under positive scalar multiplication and thus that we can restrict ourselves to effects. Let $a \in F$ be an effect. By Corollary 24, we can write $a=\lambda r+\mu r^{\perp}$ for some $\lambda, \mu \geq 0$ and $r$ atomic. Suppose both $\lambda, \mu>0$, then $F$ is a face, $r, r^{\perp} \in F$ so that $\frac{1}{2} 1=\frac{1}{2}\left(r+r^{\perp}\right) \in F$. But then since $1=s$ $+s^{\perp}$ for any atomic $s$, we see that $F$ has to be the entire positive cone. We conclude that we must have had $a=\lambda r$ for some atomic $r$. If there were some other atomic $s \in F$, then we can consider $a=\frac{1}{2}(r+s)$. We know that $a$ cannot be atomic, so we can write it as $a=\lambda r+\mu r^{\perp}$ with $\lambda$, $\mu>0$ which is a contradiction. We conclude that $F$ is an extreme ray and thus that the positive cone of $V_{p, q}$ is strictly convex.

Corollary 27. Let $p$ and $q$ be two different atomic effects, then $V_{p, q}$ is isomorphic to a spin-factor.

Proof. Follows directly from the previous lemma and Proposition 25.

Recall that a state on an order unit space is a positive linear map $\omega: V \rightarrow \mathbb{R}$ such that $\omega(1)=1$. For an atomic effect $p$ in a spin-factor, there exists a unique state $\omega_{p}$ such that $\omega_{p}(p)=1$. A spin-factor has symmetry of transition probabilities: ${ }^{23} \omega_{p}(q)=\omega_{q}(p)$ for any two atomic effects $p$ and $q$. We can use the previous results to prove that symmetry of transition probabilities also holds for arbitrary (finite-dimensional) sequential product spaces.

Proposition 28. Let $p, q \in E$ be atomic effects. There exist unique pure states $\omega_{p}$ and $\omega_{q}$ such that $\omega_{p}(p)=1$ and $\omega_{q}(q)=1$. Furthermore, for these states, we have $\omega_{p}(q)=\omega_{q}(p)$.

Proof. The states separate the points of an order unit space (Corollary 1.27 of Ref. 22) so that for $p$ we can find a state $\omega$ such that $\omega(p)$ $\neq 0$. Define $\omega_{p}(a):=\omega(p \& a) /(\omega(p))$, then $\omega_{p}$ is a state and $\omega_{p}(p)=1$. Suppose there is another state $\omega^{\prime}$ such that $\omega^{\prime}(p)=1$. Let $q \neq p$ be any other atomic effect (if there is no atomic $q \neq p$, then $V \cong \mathbb{R}$ and we are already done) and look at the restrictions of the states $\omega_{p}$ and $\omega^{\prime}$ to the space $V_{p, q}$. These restriction maps are still states as $\omega_{p}(p \vee q) \geq \omega_{p}(p)=1$ (and similarly for $\left.\omega^{\prime}\right)$. Because states with this property are unique on spin-factors, we can conclude that these restricted states are equal on this subspace and, in particular, $\omega_{p}(q)=\omega^{\prime}(q)$. Since $q$ was arbitrary and the atomic effects span $V$, we conclude that $\omega_{p}=\omega^{\prime}$ so that $\omega_{p}$ is indeed unique.

For any two atomic $p$ and $q$, we can look at their unique pure states $\omega_{p}$ and $\omega_{q}$ as restricted to $V_{p, q}$ for which we know that $\omega_{p}(q)=\omega_{q}(p)$ which finishes the proof.

Proposition 29. Let $p$ and $q$ be atomic sharp effects. $p$ and $q$ are orthogonal, i.e., $p \& q=q \& p=0$ if and only if $\omega_{p}(q)=\omega_{q}(p)=0$. Furthermore, $p \& q=\omega_{p}(q) p$.

Proof. Note that if $q \& p=0$, then by Proposition $12.1 q+p \leq 1$ so that $1=\omega(p) \leq \omega_{p}(q+p) \leq \omega(1)=1$ from which we conclude that $\omega_{p}(q)=0$. So, if $p$ and $q$ are orthogonal, then $\omega_{p}(q)=\omega_{q}(p)=0$.

For the converse, we will show that $p \& q=\omega_{p}(q) p$, from which it directly follows that $\omega_{p}(q)=0 \Rightarrow p \& q=0$. Since $p$ is atomic, we of course have $p \& q=\lambda p$ for some $\lambda \geq 0$. Let $\omega^{\prime}(a)=\omega_{p}(p \& a)$, then $\omega^{\prime}(p)=\omega_{p}(p \& p)=\omega_{p}(p)=1$ so that by the uniqueness of $\omega_{p}$ we have $\omega^{\prime}=\omega_{p}$. We then see that $\omega_{p}(q)=\omega^{\prime}(q)=\omega_{p}(p \& q)=\omega_{p}(\lambda p)=\lambda \omega_{p}(p)=\lambda$. 
Proposition 30. There exists an inner product on $V$ such that the positive cone is self-dual with respect to this inner product.

Proof. For atomic $p$ and $q$, we let $\langle p, q\rangle:=\omega_{p}(q)=\omega_{q}(p)=\langle q, p\rangle$. We can then extend it by linearity to arbitrary $a=\sum_{i} \lambda_{i} p_{i}$ and $b=\sum_{j} \mu_{j} q_{j}$ by $\langle a, b\rangle:=\sum_{i, j} \lambda_{i} \mu_{j}\left\langle p_{i}, q_{j}\right\rangle$. This is well-defined since $\langle a, b\rangle=\sum_{i} \lambda_{i} \omega_{p_{i}}\left(\sum_{j} \mu_{j} q_{j}\right)=\sum_{i} \lambda_{i} \omega_{p_{i}}(b)=\sum_{j} \mu_{j} \omega_{q_{j}}(a)$ so that this is independent of the representation of $a$ and $b$ as linear combinations of atomic effects. Now, $\langle a, a\rangle=\sum_{i, j} \lambda_{i} \lambda_{j} \omega_{p_{i}}\left(p_{j}\right)=\sum_{i} \lambda_{i}^{2}$ since $p_{i}$ and $p_{j}$ are orthogonal when $i \neq j$ and $\omega_{p_{i}}\left(p_{i}\right)=1$. We conclude that $\langle a, a\rangle \geq 0$ and that it is only equal to zero when $a=0$ so that $\langle\cdot, \cdot\rangle$ indeed is an inner product.

If $a$ and $b$ are positive elements, then we can write them as $a=\sum_{i} \lambda_{i} p_{i}$ and $b=\sum_{j} \mu_{j} q_{j}$, where all $\lambda_{i}$ and $\mu_{j}$ are greater than zero. It then easily follows that $\langle a, b\rangle \geq 0$ because $\omega_{p_{i}}\left(q_{j}\right) \geq 0$. Conversely, if we have $a=\sum_{i} \lambda_{i} p_{i}$ with $\lambda_{i}$ not necessarily positive with $\langle a, b\rangle \geq 0$ for all $b \geq 0$, then we can, in particular, take $b=p_{j}$ to see that $0 \leq\left\langle a, p_{j}\right\rangle=\lambda_{j}$ from which we conclude that indeed $a \geq 0$.

Theorem 1. A finite-dimensional sequential product space is isomorphic to a Euclidean Jordan algebra.

Proof. By Proposition 8, the space is homogeneous, and by the previous proposition, it is self-dual. The Koecher-Vinberg theorem ${ }^{2}$ states that any finite-dimensional homogeneous self-dual ordered vector space is order-isomorphic to a Euclidean Jordan algebra.

\section{THE JORDAN PRODUCT FROM A SEQUENTIAL PRODUCT}

The Koecher-Vinberg theorem is a rather indirect way of establishing the Jordan algebra structure of the space. Since we do not have just a homogeneous self-dual space, but we also have access to the sequential product, we can in fact construct the Jordan product directly. That is what we will strive for in this section. We will use the construction of the Jordan product from the work of Alfsen and Shultz ${ }^{23}$ but then adapted to our setting. In this section, we will again let $V$ be a finite-dimensional sequential product space, and hence, by Sec. III D, it is self-dual and homogeneous. We write $L_{a}: V \rightarrow V$ for the linear map that sends $v \in V$ to $a \& v$.

Definition 15. Let $p$ be an atomic sharp effect, and let $b \in V$ be arbitrary. We define their Jordan product as $p * b=\frac{1}{2}\left(\mathrm{id}+L_{p}-L_{p^{\perp}}\right) b$.

Lemma 31. Let $p$ and $q$ be atomic sharp effects, then $p^{\perp} \& q=p^{\prime} \& q$, where $p^{\prime}=p \vee q-p$.

Proof. First, note that $p^{\perp}=1-p=1-p \vee q+p \vee q-p=(p \vee q)^{\perp}+p^{\prime}$ and hence that $p^{\prime} \leq p^{\perp}$ so that $p^{\prime} \mid p^{\perp}$ by Proposition 9.1. We then also have $p^{\perp} \&(p \vee q)=(p \vee q) \& p^{\perp}=(p \vee q) \&\left((p \vee q)^{\perp}+p^{\prime}\right)=p^{\prime}$. Now, using the fact that we are working with compatible effects and that $q \leq p \vee q$, we calculate $p^{\perp} \& q=p^{\perp} \&((p \vee q) \& q)=\left(p^{\perp} \&(p \vee q)\right) \& q=p^{\prime} \& q$.

Lemma 32 (Lemma 9.29 of Ref. 23): Let $p$ and $q$ be sharp atomic effects.

1. $p * q=q * p$.

2. When $p \& q=0$, we have $p * q=0$, and in that case, for any $b \in V, p *(q * b)=q *(p * b)$.

3. $p * p=p$.

Proof.

1. If $p=q$ this is trivial, so assume that $p \neq q$. Let us denote $p^{\prime}=p \vee q-p$. By Proposition $22, p^{\prime}$ is atomic. By Proposition 29 , we have $p \& q$ $=\omega_{p}(q) p=\langle p, q\rangle p$ and similarly $p^{\prime} \& q=\left\langle p^{\prime}, q\right\rangle p^{\prime}$. Expanding the definition of $p * q$ and using lemma 31 to write $p^{\perp} \& q=p^{\prime} \& q$, where $p^{\prime}=p \vee q-p$, we calculate

$$
\begin{aligned}
2(p * q) & =q+\langle p, q\rangle p-\left\langle p^{\prime}, q\right\rangle p^{\prime} \\
& =q+\langle p, q\rangle p-\left\langle p^{\prime}, q\right\rangle(p \vee q-p) \\
& =q+\left(\langle p, q\rangle p+\left\langle p^{\prime}, q\right\rangle\right) p-\left\langle p^{\prime}, q\right\rangle(p \vee q) \\
& =q+\langle p \vee q, q\rangle p+\langle p \vee q-p, q\rangle(p \vee q) \\
& =q+p+(1-\langle p, q\rangle)(p \vee q),
\end{aligned}
$$

which is indeed symmetric in $p$ and $q$.

2. When $p \& q=0$, we have $q \leq p^{\perp}$ so that $p^{\perp} \& q=q$ which indeed gives $p * q=\frac{1}{2}\left(q+p \& q-p^{\perp} \& q\right)=\frac{1}{2}(q-q)=0$. For the second point, we note that because $p \& q=0$ we have $p \mid q, q^{\perp}$ and $q \mid p^{\perp}$, and hence that the maps $L_{p}, L_{p^{\perp}}, L_{q}$ and $L_{q^{\perp}}$ commute so that the maps $b \mapsto p * b$ and $b \mapsto q * b$ will commute as well.

3. Follows immediately from $p \& p=p$ and $p^{\perp} \& p=0$.

As a result of this lemma, we can extend the Jordan product by linearity to the entirety of the space. 
Definition 16. Let $a, b \in V$ be arbitrary. Let $a=\sum_{i} \lambda_{i} p_{i}$ and $b=\sum_{j} \mu_{j} q_{j}$ be spectral decompositions with $p_{i}$ and $q_{j}$ atomic. Define their Jordan product as $a * b=\sum_{i, j} \lambda_{i} \mu_{j}\left(p_{i} * q_{j}\right)$. We write $T_{a}: V \rightarrow V$ for the operator that sends $b$ to $a * b$.

Proposition 33. The Jordan product is well-defined, bilinear, and commutative, and furthermore,

1. if $a \mid b$, then $T_{a} T_{b}=T_{b} T_{a}$, and

2. If $a \mid b$, then $T_{a} b=a^{+} \& b-a^{-} \& b$, where $a^{+}$and $a^{-}$are the unique orthogonal positive elements such that $a=a^{+}-a^{-}$.

Proof. Write $a$ as a spectral decomposition into atomic effects: $a=\sum_{i} \lambda_{i} p_{i}$. We first note that we of course have $a * b=\sum_{i} \lambda_{i} p_{i} * b$ so that the definition is independent of how $b$ is represented as a sum of atomic sharp effects. By the previous lemma, the product is commutative, and therefore, we see it is bilinear and well-defined.

1. Suppose $a \mid b$. By considering the classical algebra spanned by both $a$ and $b$, we can find an orthogonal set of atomic sharp effects $p_{i}$ such that $a=\sum_{i} \lambda_{i} p_{i}$ and $b=\sum_{i} \mu_{i} p_{i}$. Since $p_{i} * p_{j}=0$ for $i \neq j$, we have $p_{i} *\left(c * p_{j}\right)=p_{j} *\left(c * p_{i}\right)$ by Lemma 32 so that we can then write

$$
T_{b} T_{a} c=b *(a * c)=\sum_{i, j} \mu_{i} \lambda_{j} p_{i} *\left(c * p_{j}\right)=\sum_{i, j} \mu_{i} \lambda_{j} p_{j} *\left(c * p_{i}\right)=a *(c * b)=T_{a} T_{b} c
$$

which holds for all $c$. We conclude that $T_{b} T_{a}=T_{a} T_{b}$.

2. If atomic $p_{i}$ commutes with $b$, then $p_{i} * b=\frac{1}{2}\left(\mathrm{id}+U_{p_{i}}-U_{p_{i}^{\perp}}\right) b=\frac{1}{2}\left(b+p_{i} \& b-p_{i}^{\perp} b\right)=\frac{1}{2}\left(\left(p_{i}+p_{i}^{\perp}\right) \& b+p_{i} \& b-p_{i}^{\perp} b\right)=p_{i} \& b$. So by writing $a=\sum_{i} \lambda_{i} p_{i}=\sum_{i, \lambda_{i}>0} \lambda p_{i}-\sum_{i, \lambda_{i}<0}\left|\lambda_{i}\right| p_{i}=a^{+}-a^{-}$with $p_{i} \mid b$, the desired result follows by linearity.

Theorem 2. Let $V$ be a finite-dimensional sequential product space, then it is a Euclidean Jordan algebra with the Jordan product as defined above.

Proof. We have already established that the product is bilinear and commutative. Note that since $a \mid a$ we get $a * a=a^{+} \& a-a^{-} \& a=$ $\left(a^{+}\right)^{2}+\left(a^{-}\right)^{2}=a^{2}$ using Proposition 33.2. Because of course $a \mid a^{2}$, we get $T_{a} T_{a * a}=T_{a * a} T_{a}$ as a consequence of Proposition 33.1 so that the Jordan identity holds. Since $a * a=a^{2} \geq 0$, we also see that the algebra is formally real: if $\sum_{i} a_{i} * a_{i}=0$, then for all $i$, $a_{i}=0$. It is a well-known result [see, for instance, Ref. 27 (Proposition VIII.4.2)] that if a Jordan product is formally real, the algebra is Euclidean, with the product being symmetric with regard to the (essentially unique) self-dual inner product.

Remark 34. It is also possible to show in a more direct manner that the Jordan product is symmetric with respect to the inner product. We sketch here how to do so. First, it must be established that $L_{a}$ for invertible $a$ commute with their adjoints $L_{a}^{*}$ by exploiting the fact that $\Theta=L_{a^{-1}} L_{a}^{*}$ must be a unital order-isomorphism necessarily satisfying $\Theta^{-1}=\Theta^{*}$. Because the mapping $a \mapsto L_{a}$ is continuous, the result extends to all $a$, and hence, it holds, in particular, for $L_{p}$ with $p$ sharp. It is a standard result that an idempotent map that commutes with its adjoint is in fact self-adjoint. Since the Jordan product is defined as a linear combination of product maps of sharp effects, this indeed establishes the desired result.

\section{LOCAL TOMOGRAPHY AND C* -ALGEBRAS}

In this section, we will let $V$ and $W$ be finite-dimensional sequential product spaces, and hence, in Secs. III and IV, they will be Euclidean Jordan algebras. For the duration of this section, we will assume that they have a locally tomographic composite (see Definition 4), i.e., that their linear algebraic tensor product $V \otimes W$ is also a sequential product space and that the sequential product satisfies

$$
\left(a_{1} \otimes b_{1}\right) \&\left(a_{2} \otimes b_{2}\right)=\left(a_{1} \& a_{2}\right) \otimes\left(b_{1} \& b_{2}\right)
$$

Note that by the definition of the tensor product, any element of $V \otimes W$ can be written as $\sum_{i} \lambda_{i} a_{i} \otimes b_{i}$, where $a_{i} \in V, b_{i} \in W$, and $\lambda_{i} \in \mathbb{R}$.

Proposition 35. Let $p \in V$ and $q \in W$ be atomic, then $p \otimes q \in V \otimes W$ is also atomic.

Proof. Because $(p \otimes q) \&(p \otimes q)=(p \& p) \otimes(q \& q)=p \otimes q$, it is sharp. Let $c=\sum_{i} \lambda_{i} a_{i} \otimes b_{i}$ be an arbitrary element of $V \otimes W$, then using Lemma 17, $(p \otimes q) \& c=\sum_{i} \lambda_{i}\left(p \& a_{i}\right) \otimes\left(q \& b_{i}\right)=\sum_{i} \lambda_{i}\left\|p \& a_{i}\right\|\left\|q \& b_{i}\right\|(p \otimes q)=\mu(p \otimes q)$ for some $\mu \in \mathbb{R}$. Since $c$ was arbitrary, we conclude that $p \otimes q$ is atomic as a result of Lemma 17 .

Definition 17. Let $V$ be a sequential product space with effects $E$. We call $c \in V$ classical when it is sharp and compatible with all other effects: $a \mid c$ for all $a \in E$. We will call a classical effect minimal when there is no nonzero classical effect strictly below it. 
Proposition 36. Let $c \in V$ and $d \in W$ be classical, then $c \otimes d$ is classical in $V \otimes W$.

Proof. $c \mid a$ for all $a \in V$ and $d \mid b$ for all $b \in W$; therefore, $c \otimes d \mid a \otimes b$ and the same holds for linear combinations of these elements which span the entirety of $V \otimes W$.

We call an EJA simple if it contains no nontrivial classical effects. We can write any EJA uniquely as $E_{1} \oplus \ldots \oplus E_{k}$, where $E_{i}$ are simple EJAs, which we will refer to as the summands of the EJA. An EJA with $k$ summands has exactly $k$ minimal classical effects, corresponding to the units of each of the summands. Each other classical effect is a sum of these minimal ones.

Lemma 37. Let $p$ and $q$ be atomic effects in an EJA. If $q \& p \neq 0$, then $p$ and $q$ belong to the same simple summand.

Proof. Suppose $c$ is a sharp classical effect. Then, $c \& p=p \& c=\lambda p$, for some $\lambda \in[0,1]$. But also $\lambda p=c \& p=\left(c^{2}\right) \& p=c \&(c \& p)=\lambda(c$ $\& p)=\lambda^{2} p$ so that $\lambda=1$ or $\lambda=0$. So, either $p \leq c$ or $p$ and $c$ are orthogonal. Suppose $p \leq c$ and that $q \& p \neq 0$, then $0 \neq q \& p \leq q \& c=\lambda q$ so that $\lambda \neq 0$ and thus $q \leq c$. If we let $c$ be the identity of the summand that $p$ belongs to, we see that the desired property follows.

Lemma 38. Let $p_{1}, \ldots, p_{r}$ be a maximal collection of orthogonal nonzero atomic effects in a simple EJA, then there exists an atomic effect $q$ such that $q \& p_{i} \neq 0$ for all $i$.

Proof. We do this by case distinction using the classification of simple EJAs. ${ }^{1}$ The space is a spin-factor, in which case a maximal collection is always given by a sharp atomic $p$ and its complement $p^{\perp}$. Any $q \neq p, p^{\perp}$ cannot be orthogonal to them, because the space is of rank 2 , so that indeed $q \& p \neq 0$ and $q \& p^{\perp} \neq 0$.

If the space is not a spin-factor, then it must be of the form $B(H)^{\text {sa }}$ for a real, complex, quaternionic, or octonion finite-dimensional Hilbert space $H$ (in the case of the octonions, we must have $\operatorname{dim} H=3$ ). For such a space, the atomic idempotents correspond to unit vectors of the underlying Hilbert space: $p_{i}=\left|v_{i}\right\rangle\left\langle v_{i}\right|$, where $v_{i} \in H$ is some unit vector. We can then take $q=|w\rangle\langle w|$ with $w=\frac{1}{\sqrt{r}} \sum_{i=1}^{r} v_{i}$. It should then be clear that $\left\langle q, p_{i}\right\rangle \neq 0$ and hence $q \& p_{i} \neq 0$.

Proposition 39. Let $V=E_{1} \oplus \ldots \oplus E_{m}$ and $W=F_{1} \oplus \ldots \oplus F_{n}$ with $E_{i}$ and $F_{j}$ being simple EJAs. Let $1 \leq k \leq n$ and $1 \leq l \leq m$. Let $p_{1}, \ldots, p_{r}$ be a maximal collection of orthogonal nonzero atomic effects in $E_{k}$, and let $q_{1}, \ldots, q_{s}$ be such a maximal collection in $F_{l}$. Then, $\left(p_{i} \otimes q_{j}\right)_{i=1, j=1}^{r, s}$ belong to the same simple summand in $V \otimes W$ and they form a maximal collection of orthogonal nonzero atomic effects in this summand.

Proof. We let $p$ be atomic such that $p \& p_{i} \neq 0$ for all $i$ which exists by the previous lemma, and similarly, we let $q$ be atomic such that $q$ $\& q_{j} \neq 0$ for all $j$. By Proposition 35, $p \otimes q$ and $p_{i} \otimes q_{j}$ will be atomic for all $i$ and $j$. By construction, we of course have $0 \neq\left(p \& p_{i}\right) \otimes\left(q \& q_{j}\right)=$ $(p \otimes q) \&\left(p_{i} \otimes q_{j}\right)$, and by Lemma 37, $p_{i} \otimes q_{j}$ must then belong to the summand of $p \otimes q$ for all $i$ and $j$.

Since $\sum_{i} p_{i}=1_{E_{k}}$, this sum is a classical effect. The same holds for $\sum_{j} q_{j}=1_{F_{l}}$. Their tensor product $1_{E_{k}} \otimes 1_{F_{l}}=\sum_{i, j} p_{i} \otimes q_{j}$ is then also classical by Proposition 36. Since the only nonzero classical effect in a simple summand is the identity, this expression must be equal to the identity of this summand. As a result, the set $\left(p_{i} \otimes q_{j}\right)_{i, j}$ is indeed maximal.

Using this proposition, we conclude that for each of the summands $E$ of $V$ and $F$ of $W$, there must exist a summand in $V \otimes W$ which has $\operatorname{rank} \operatorname{rnk} E \operatorname{rnk} F$. Because the tensor product map is obviously injective, this factor must have dimension at least $\operatorname{dim} E \operatorname{dim} F$, then because of local tomography, the dimension must be strictly equal. Now, let $V$ be a sequential product space for which the tensor product $V \otimes V$ exists so that the above must, in particular, be true when $E=F$, i.e., if $E$ is a simple factor of $V$, then there must exist a simple factor with rank (rnkE) ${ }^{2}$ and dimension $(\operatorname{dim} E)^{2}$.

Proposition 40. Let $E$ be a simple Euclidean Jordan algebra of rank $r>1$ and dimension $N$. There exists a simple Euclidean Jordan algebra of rank $r^{2}$ and dimension $N^{2}$ if and only if $E=B(H)^{\mathrm{sa}}$, where $H$ is a complex finite-dimensional Hilbert space.

Proof. If $E=B(H)^{\mathrm{sa}}$ is a complex matrix algebra, the property is obviously true by considering $B(H \otimes H)^{\text {sa }}$ as the simple EJA of rank $r^{2}$ and dimension $N^{2}$. We simply check that every other simple EJA is not a possibility. If $E=B(H)^{\mathrm{sa}}$, where $H$ is the 3 -dimensional octonion Hilbert space, then $r=3$ and $N=27$. The highest dimensional simple EJA of rank 9 is the quaternionic system which has dimension $9 *(2 *$ $9-1)=153<27^{2}=729$ so that this is not possible. If $E=B(H)^{\text {sa }}$ with $H$ quaternionic, then $N=r(2 r-1)$. The highest dimensional simple EJA of rank $r^{2}$ is also quaternionic so that its dimension is $r^{2}\left(2 r^{2}-1\right)$. It is easy to check that $N^{2}=r^{2}(2 r-1)^{2}>r^{2}\left(2 r^{2}-1\right)$ when $r>1$ so that again it cannot be this space. If $E=B(H)^{\text {sa }}$, where $H$ is real, then by dimension counting we can again see that there does not exist an EJA with rank $r^{2}$ and dimension $N^{2}$. A spin factor always has rank 2. The rank 4 EJAs have dimension 10, 16, and 28 . The only one of these which is a square is 16 . The 4 dimensional spin-factor corresponds to the qubit which is indeed $B(H)^{\text {sa }}$ with $H$ as a 2-dimensional complex Hilbert space. 
Theorem 3. Suppose $V$ is a finite-dimensional sequential product space which has a locally tomographic composite with itself, i.e., for which the linear algebraic tensor product $V \otimes V$ is also a sequential product space, satisfying $(a \otimes b) \&(c \otimes d)=(a \& c) \otimes(b \& d)$ for all $a, b$, $c, d \in V$. Then, there exists a $C^{*}$-algebra $A$ such that $V \cong A^{\text {sa }}$.

Proof. As established, $V$ is a Euclidean Jordan algebra. As a result of Proposition 39, for each summand of $V=E_{1} \oplus \ldots \oplus E_{n}$, there must exist a simple EJA of rank $\left(\operatorname{rnk} E_{i}\right)^{2}$ and dimension $\left(\operatorname{dim} E_{i}\right)^{2}$. By Proposition 40 , this is only possible if $E_{i}=B(H)^{\mathrm{sa}}$, where $H$ is a complex Hilbert space. Therefore, $V$ is a direct sum of complex matrix algebras which means it is the set of self-adjoint elements of a $\mathrm{C}^{*}$-algebra.

This result should be compared to a result by Hanche-Olsen that states that when $V$ is a JB-algebra, such that $V \otimes B\left(\mathbb{C}^{2}\right)^{\text {sa }}$ is also a JB-algebra satisfying $(a \otimes b) *\left(c^{*} \otimes d\right)=(a * c) \otimes(b * d), V$ must be the self-adjoint part of a $C^{*}$-algebra. ${ }^{20}$

\section{INFINITE-DIMENSIONAL SEQUENTIAL PRODUCT SPACES}

In order to state an infinite-dimensional generalization of Theorem 2, we must first give an appropriate definition of infinite-dimensional Jordan algebras.

Definition 18. An order unit space $V$ is a JB-algebra when it is complete in its norm topology, and it has a Jordan product $*$ such that $\|a * a\|=\|a\|^{2}$ and $\|a * b\| \leq\|a\|\|b\|$ for all $a, b \in V$.

As the proof in the finite-dimensional case relied heavily on using atomic effects, which do not have to exist in infinite dimension, we will need to require a few other properties. In particular, we will assume that the order structure on the space is quite rich:

Definition 19. Let $V$ be an order unit space. A subset $S \subseteq V$ is called bounded when there exists $r \in \mathbb{R}$ such that $\|a\| \leq r$ for all $a \in S$. We say $V$ is $\sigma$-complete when every bounded increasing sequence $a_{1} \leq a_{2} \leq a_{3} \leq \ldots$ has a supremum.

Definition 20. A state $\omega: V \rightarrow \mathbb{R}$ on an order unit space $V$ is called $\sigma$-normal when it preserves suprema of bounded increasing sequences: $\omega\left(\vee_{i} a_{i}\right)=\vee_{i} \omega\left(a_{i}\right)$. We say that $V$ has enough normal states when the $\sigma$-normal states separate the elements, i.e., when $\omega(v)=\omega(w)$ for all $\sigma$-normal states, $\omega$ implies that $v=w$.

Finally, we slightly modify the definition of sequential product in order to be more aligned to this order structure:

Definition 21. A $\sigma$-sequential product space is a (possibly infinite-dimensional) $\sigma$-complete order unit space $V$ with enough normal states that comes equipped with a map $\&:[0,1]_{V} \times[0,1]_{V} \rightarrow[0,1]_{V}$ satisfying for all $a, b, c \in[0,1]_{V}$ :

(T1) Additivity: $a \&(b+c)=a \& b+a \& c$.

(T2) Normality: The map $b \mapsto a \& b$ is $\sigma$-normal: $a \&\left(\vee_{i} b_{i}\right)=\vee_{i}\left(a \& b_{i}\right)$ for an increasing sequence $\left\{b_{i}\right\}$.

(T3) Unitality: $1 \& a=a$.

(T4) Compatibility of orthogonal effects: If $a \& b=0$, then also $b \& a=0$.

(T5) Associativity of compatible effects: If $a \mid b$, then $a \&(b \& c)=(a \& b) \& c$.

(T6) Additivity of compatible effects: If $a \mid b$, then $a \mid 1-b$, and if also $a \mid c$, then $a \mid(b+c)$.

(T7) Multiplicativity of compatible effects: If $a \mid b$ and $a \mid c$, then $a \mid(b \& c)$.

We call this map the sequential product of the space.

Remark 41. The definition of the sequential product operation is exactly the one of Definition 2, except that we require the operation to be normal in the second argument, instead of being norm-continuous in the first argument. The definition here corresponds exactly to the $\sigma$-sequential product of Ref. 11 .

For the remainder of the section, we will let $V$ denote a $\sigma$-sequential product space and \& denote its sequential product. We conjecture that each $\sigma$-sequential product space is a JB-algebra, but we do not seem to have the right tools to prove this. In order to show a correspondence of $\sigma$-sequential product spaces to $\sigma$-complete JB-algebras, we will need to assume two additional properties on the sequential product.

The first is inspired by the notion of comprehension from effectus theory: $:^{28,29}$

Definition 22. We say the sequential product is comprehensive when for all sharp effects $q$ the following implication holds for all $\sigma$ normal states $\omega$ : if $\omega(q)=1$, then $\omega(q \& p)=\omega(p)$ for any $p$. 
What this property says is that if an effect $q$ already holds with certainty on a state $\omega$, then measuring $q$ does not affect the probabilities of other effects holding in the state $\omega$. If the sequential product of $V$ is comprehensive, then every effect of $V$ has a comprehension, as defined in Ref. 28, hence the name.

The second property is a weaker version of the fundamental equality of quadratic Jordan algebras. ${ }^{30}$

Definition 23. We say the sequential product is quadratic when for any two sharp effects $p$ and $q$ we have $q \&(p \& q)=(q \& p)^{2}$.

We unfortunately do not know of a reasonable operational interpretation of this property, but we do note that if we are considering $\mathrm{C}^{*}$-algebras, then this property is evidently true because $q \&(p \& q)=q(p q p) q$ while $(q \& p)^{2}=(q p q)^{2}$. This property also naturally arises in a $\dagger$-effectus. ${ }^{31}$

We will now work toward showing that $\sigma$-sequential product spaces with a comprehensive quadratic sequential product are JB-algebras. Our proof is based on Theorem 9.84 of Ref. 23.

Definition 24. We call $a \in V$ simple when we can write it as $a=\sum_{i=1}^{r} \lambda_{i} p_{i}$ for some $r \in \mathbb{N}$, where $\lambda_{i} \in \mathbb{R}$ and $p_{i}$ are sharp and orthogonal.

Proposition 42 (Ref. 16). Every element $a \in V$ of a $\sigma$-sequential product space $V$ can be written as the supremum of an increasing sequence of simple elements: $a=\vee_{i} a_{i}$, where $a_{i}$ are simple.

Lemma 43. If $a \mid b$, then $a^{2}-b^{2}=(a+b) \&(a-b)$.

Proof. We simply calculate $(a+b) \&(a-b)=(a+b) \& a-(a+b) \& b=a \&(a+b)-b \&(a+b)=a \& a+b \& a-a \& b-b \& b$ $=a \& a-b \& b$.

Lemma 44. Let $q$ and $p$ be sharp effects, then $(q \& p)^{2}-\left(q \& p^{\perp}\right)^{2}=q \& p-q \& p^{\perp}$.

Proof. Since $q \& p \mid q$ and $q \& p^{\perp}=q-q \& p$, we see that $q \& p \mid q \& p^{\perp}$, and hence, by the previous lemma where $a=q \& p$ and $b=q \&$ $p^{\perp}$, we get $(q \& p)^{2}-\left(q \& p^{\perp}\right)^{2}=\left(q \& p+q \& p^{\perp}\right) \&\left(q \& p-q \& p^{\perp}\right)=q \&\left(q \&\left(p-p^{\perp}\right)\right)=q \& p-q \& p^{\perp}$.

Proposition 45. Suppose the sequential product is comprehensive and quadratic, then for any sharp effect $q$, the following implication holds: if $\omega(q)=0$, then $\omega(p \& q)=\omega\left(p^{\perp} \& q\right)$ for any sharp effect $p$.

Proof. Suppose $\omega(q)=0$, then of course $\omega\left(q^{\perp}\right)=1$. Note that it suffices to prove the equality $q^{\perp} \&(p \& q)=q^{\perp} \&\left(p^{\perp} \& q\right)$ since then, by using the comprehension property, $\omega(p \& q)=\omega\left(q^{\perp} \&(p \& q)\right)=\omega\left(q^{\perp} \&\left(p^{\perp} \& q\right)\right)=\omega\left(p^{\perp} \& q\right)$. By adding the expression $q^{\perp} \&\left(p \& q^{\perp}\right)+q^{\perp}$ $\&\left(p^{\perp} \& q^{\perp}\right)$ to both sides of the equality, we see that it is equivalent to proving the equality

$$
q^{\perp} \&\left(p \&\left(q+q^{\perp}\right)\right)+q^{\perp} \&\left(p^{\perp} \& q^{\perp}\right)=q^{\perp} \&\left(p^{\perp} \&\left(q+q^{\perp}\right)\right)+q^{\perp} \&\left(p \& q^{\perp}\right) .
$$

We then use the quadratic property to write $q^{\perp} \&\left(p^{\perp} \& q^{\perp}\right)=\left(q^{\perp} \& p^{\perp}\right)^{2}$ and similarly with $p^{\perp}$ replaced with $p$ to simplify this expression to

$$
q^{\perp} \& p+\left(q^{\perp} \& p^{\perp}\right)^{2}=q^{\perp} \& p^{\perp}+\left(q^{\perp} \& p\right)^{2}
$$

The desired equality is therefore proven if we can show that

$$
\left(q^{\perp} \& p\right)^{2}-\left(q^{\perp} \& p^{\perp}\right)^{2}=q^{\perp} \& p-q^{\perp} \& p^{\perp}
$$

but this follows immediately from the previous lemma.

Proposition 46. Suppose the sequential product is comprehensive and quadratic, then for any $a \geq 0$, the following implication holds for any $\sigma$-normal $\omega$ : if $\omega(a)=0$, then $\omega(p \& a)=\omega\left(p^{\perp} \& a\right)$ for any sharp effect $p$.

Proof. First, suppose $a$ is simple: $a=\sum_{i} \lambda_{i} q_{i}$. Since $\omega(a)=0$, we of course also have $\omega\left(q_{i}\right)=0$, and hence, by the previous proposition, $\omega(p$ $\left.\& q_{i}\right)=\omega\left(p^{\perp} \& q_{i}\right)$. Then, by linearity, $\omega(p \& a)=\sum_{i} \lambda_{i} \omega\left(p \& q_{i}\right)=\sum_{i} \lambda_{i} \omega\left(p^{\perp} \& q_{i}\right)=\omega\left(p^{\perp} \& a\right)$. Any $a \geq 0$ can be written as $a=\vee_{i} a_{i}$, where $a_{i}$ are simple and $a_{i} \leq a$. Suppose $\omega(a)=0$, then also $\omega\left(a_{i}\right)=0$, and hence, $\omega(p \& a)=\omega\left(p \& \vee_{i} a_{i}\right)=\omega\left(\vee_{i} p \& a_{i}\right)=\vee_{i} \omega\left(p \& a_{i}\right)=\vee_{i} \omega\left(p^{\perp} \& a_{i}\right)=$ $\omega\left(p^{\perp} \& \vee{ }_{i} a_{i}\right)=\omega\left(p^{\perp} \& a\right)$.

Theorem 4. A $\sigma$-sequential product space with a comprehensive and quadratic sequential product is a $\sigma$-complete JB-algebra. 
Proof. We follow the proof of Ref. 23 (Theorem 9.48). Let $a$ be an effect, and let $p$ be sharp. We let $L_{p}$ denote the multiplication operator of $p$. Let $D_{p}:=L_{p}-L_{p^{\perp}}$. By the previous proposition when $\omega(a)=0$, we have $\omega\left(D_{p} a\right)=\omega(p \& a)-\omega\left(p^{\perp} \& a\right)=0$. Because $V$ is $\sigma$-complete, $V$ is also complete in the norm, ${ }^{32}$ and hence, by Ref. 22 (Proposition 1.108), the linear map $D_{p}$ is then an order derivation, i.e., the exponential map $\exp \left(t D_{p}\right)$ is an order isomorphism for any $t \in \mathbb{R}$. Using exactly the same proof as in Ref. 23 (Theorem 9.48), we can then show that for any sharp $p$ and $q$ we will have $\left[D_{p}, D_{q}\right] 1=0$ and hence also $\left[T_{p}, T_{q}\right] 1=0$, where $T_{p}:=\frac{1}{2}\left(\right.$ id $\left.+D_{p}\right)$. As $T_{p} 1=p$, this commutator being zero tells us that $T_{p} q=T_{q} p$. Hence, we can define the Jordan product of sharp effects as $p * q:=T_{p} q=T_{q} p$. As this is symmetric, and linear in one of the arguments, we can easily extend this by bilinearity to all simple elements. It is then straightforward to show that for simple elements $a * a=a^{2}$, and as a consequence, $\|a * b\| \leq\|a\|\|b\|$, and hence, this product is continuous in both arguments. We can then extend this product by continuity to the entirety of the space (as the simple elements lie dense in the space). That it satisfies the Jordan equality is shown in the same way as in Theorem 2. We then have shown that the space is a JB-algebra and it is by assumption $\sigma$-complete.

\section{MINIMALITY OF AXIOMS}

In this section, we will discuss the minimality of the conditions and the axioms needed to show that finite-dimensional sequential product spaces are Euclidean Jordan algebras. For easy reference, we copy Definition 2 here:

Definition. A map \& : $[0,1]_{V} \times[0,1]_{V} \rightarrow[0,1]_{V}$ is called a sequential product when it satisfies the following properties for all $a, b, c \in[0,1]_{V}:$

(S1) Additivity: $a \&(b+c)=a \& b+a \& c$.

(S2) Continuity: The map $a \mapsto a \& b$ is continuous in the norm.

(S3) Unitality: $1 \& a=a$.

(S4) Compatibility of orthogonal effects: If $a \& b=0$, then also $b \& a=0$.

(S5) Associativity of compatible effects: If $a \mid b$, then $a \&(b \& c)=(a \& b) \& c$.

(S6) Additivity of compatible effects: If $a \mid b$, then $a \mid 1-b$, and if also $a \mid c$, then $a \mid(b+c)$.

(S7) Multiplicativity of compatible effects: If $a \mid b$ and $a \mid c$, then $a \mid(b \& c)$.

First of all, for the proofs of the main theorems, axiom S7 is actually not needed since the following weaker version is sufficient:

Proposition 47. Suppose $a \mid b, c$ and that $b \mid c$, then $a \mid(b \& c)$.

Proof. Using axiom S5 repeatedly, $a \&(b \& c)=(a \& b) \& c=(b \& a) \& c=b \&(a \& c)=b \&(c \& a)=(b \& c) \& a$.

The reason we included axiom S7 is because it is part of the definition of a sequential effect algebra and because when defining the classical algebra of an element when working in infinite dimension, it is needed to show that the algebra is closed under multiplication.

Of the other axioms, the ones that seem less essential are S4 and S2, so it would be interesting to see what can be done without them.

To define and study the classical algebra of an effect, S4 is not needed and S2 is only needed to show that $(\lambda 1) \& a=\lambda a$. The spectral theorem and the homogeneity of the space can thus be proven without using these axioms if S3 is changed to $(\lambda 1) \& a=\lambda a$. When restricting to rank 2 spaces, axioms S1, S3, S5, and S6 are then sufficient to prove that the space is a Euclidean Jordan algebra (or specifically, a spinfactor). Since the spectral theorem is also what is needed to show that $L_{a}$ for $a$ invertible is an order-isomorphism, it should be clear that on an EJA this restricted set of axioms already greatly reduces the possible sequential-product-like maps.

Note also that using the T-algebra formalism of Vinberg ${ }^{33}$ it is possible to find an associative binary operation (see the beginning of Sec. IV of Ref. 34 for this operation) for the positive elements in any finite-dimensional homogeneous space that satisfies axioms S1, S2, S3, and S5 (and by associativity also S7), but this product does not satisfy axioms S4 and S6. This actually leads to an interesting observation: if either the proof of homogeneity in Sec. II can be shown to hold without use of axiom S6 or if a binary product on homogeneous spaces can be found that also satisfies S6, then this would give us a new characterization of homogeneous spaces. In particular, in the second case, it would show that $\mathrm{S} 4$ is the key to establishing self-duality.

When one considers more general ordered vector spaces than order unit spaces, one can find nontrivial totally ordered vector spaces that allow a commutative bilinear product and hence a sequential product. ${ }^{35}$ These spaces are pathological in the sense that they have "infinitesimal" effects, i.e., effects than cannot be distinguished using states.

\section{ACKNOWLEDGMENTS}

The author would like to thank Bas and Bram Westerbaan for all the useful and insightful conversations regarding effect algebras and order unit spaces and Alex Kolmus and Ema Alsina for suggestions to improve the manuscript. This work was supported by the ERC under the European Union's Seventh Framework Programme (No. FP7/2007-2013)/ERC Grant No. 320571. 


\section{REFERENCES}

${ }^{1}$ P. Jordan, J. v. Neumann, and E. Wigner, "On an algebraic generalization of the quantum mechanical formalism," Ann. Math. 35, 29-64 (1934).

${ }^{2}$ M. Koecher, "Positivitatsbereiche im $R^{n}$," Am. J. Math. 79, 575-596 (1957).

${ }^{3}$ L. Hardy, "Reconstructing quantum theory," in Quantum Theory: Informational Foundations and Foils (Springer, 2016), pp. 223-248.

${ }^{4} \mathrm{~J}$. van de Wetering, "An effect-theoretic reconstruction of quantum theory," preprint arXiv:1801.05798 (2018).

${ }^{5}$ J. H. Selby, C. M. Scandolo, and B. Coecke, "Reconstructing quantum theory from diagrammatic postulates," preprint arXiv:1802.00367 (2018).

${ }^{6}$ H. Barnum, M. P. Müller, and C. Ududec, "Higher-order interference and single-system postulates characterizing quantum theory," New J. Phys. 16, 123029 (2014).

7. Gunson, "On the algebraic structure of quantum mechanics," Commun. Math. Phys. 6, 262-285 (1967).

${ }^{8}$ S. Tull, "A categorical reconstruction of quantum theory," preprint arXiv:1804.02265 (2016).

${ }^{9}$ P. A. Höhn, "Toolbox for reconstructing quantum theory from rules on information acquisition," Quantum 1, 38 (2017).

${ }^{10}$ G. Chiribella, G. M. D’Ariano, and P. Perinotti, “Informational derivation of quantum theory,” Phys. Rev. A 84, 012311 (2011).

${ }^{11}$ S. Gudder and R. Greechie, "Sequential products on effect algebras," Rep. Math. Phys. 49, 87-111 (2002).

${ }^{12}$ J. Barrett, "Information processing in generalized probabilistic theories," Phys. Rev. A 75, 032304 (2007).

${ }^{13} \mathrm{~L}$. Weihua and W. Junde, "A uniqueness problem of the sequence product on operator effect algebra E(H)," J. Phys. A: Math. Theor. 42, 185206 (2009).

${ }^{14}$ S. Gudder and F. Latrémolière, "Characterization of the sequential product on quantum effects," J. Math. Phys. 49, 052106 (2008).

${ }^{15}$ A. Westerbaan and B. Westerbaan, “A universal property for sequential measurement," J. Math. Phys. 57, 092203 (2016).

${ }^{16}$ J. van de Wetering, "Three characterisations of the sequential product," J. Math. Phys. 59(8), 082202 (2018).

${ }^{17}$ P. Jordan, Über Verallgemeinerungsmöglichkeiten des Formalismus der Quantenmechanik (Weidmann, 1933).

${ }^{18}$ H. Barnum and A. Wilce, "Local tomography and the Jordan structure of quantum theory," Found. Phys. 44, 192-212 (2014).

${ }^{19}$ L. Masanes, M. P. Mueller, D. Perez-Garcia, and R. Augusiak, "Entanglement and the three-dimensionality of the Bloch ball," J. Math. Phys. 55, 122203 (2014).

${ }^{20} \mathrm{H}$. Hanche-Olsen, "JB-algebras with tensor products are C $\mathrm{C}^{*}$-algebras," in Operator Algebras and Their Connections with Topology and Ergodic Theory (Springer, 1985), pp. 223-229.

${ }^{21}$ L. Hardy, "Quantum theory from five reasonable axioms," preprint arXiv:quant-ph/0101012 (2001).

${ }^{22}$ E. M. Alfsen and F. W. Shultz, State Spaces of Operator Algebras: Basic Theory, Orientations, and C ${ }^{*}$-products (Springer Science \& Business Media, 2012).

${ }^{23}$ E. M. Alfsen and F. W. Shultz, Geometry of State Spaces of Operator Algebras (Springer Science \& Business Media, 2012).

${ }^{24} \mathrm{M}$. Ito and B. F. Lourenço, “The $p$-cones in dimension $n \geq 3$ are not homogeneous when $p \neq 2$," Linear Algebra Appl. 533, 326-335 (2017).

${ }^{25}$ S. Gudder and R. Greechie, "Uniqueness and order in sequential effect algebras," Int. J. Theor. Phys. 44, 755-770 (2005).

${ }^{26} \mathrm{R}$. V. Kadison, A Representation Theory for Commutative Topological Algebra (American Mathematical Society, 1951), Vol. 7.

${ }^{27}$ J. Faraut and A. Korányi, Analysis on Symmetric Cones (Clarendon Press Oxford, 1994).

${ }^{28}$ K. Cho, B. Jacobs, B. Westerbaan, and A. Westerbaan, “An introduction to effectus theory," preprint arXiv:1512.05813 (2015).

${ }^{29}$ K. Cho, B. Jacobs, B. Westerbaan, and B. Westerbaan, "Quotient-comprehension chains," in Electronic Proceedings in Theoretical Computer Science, Proceedings of the 12th International Workshop on Quantum Physics and Logic, Oxford, U.K., July 15-17, 2015, edited by C. Heunen, P. Selinger, and J. Vicary (Open Publishing Association, 2015), Vol. 195, pp. 136-147.

${ }^{30}$ K. McCrimmon, “A general theory of Jordan rings," Proc. Natl. Acad. Sci. U. S. A. 56, 1072-1079 (1966).

${ }^{31}$ B. Westerbaan, "Dagger and dilations in the category of von Neumann algebras," Ph.D. thesis, Radboud Universiteit Nijmegen, 2018.

${ }^{32}$ B. Jacobs and A. Westerbaan, "Distances between states and between predicates," preprint arXiv:1711.09740 (2017).

${ }^{33}$ E. B. Vinberg, "Theory of homogeneous convex cones," Trans. Moscow Math. Soc. 12, 303-368 (1967).

${ }^{34}$ C. B. Chua, "Relating homogeneous cones and positive definite cones via T-algebras," SIAM J. Control 14, 500-506 (2003).

${ }^{35}$ B. Westerbaan, "Sequential product on effect logics," M.S. thesis, Radboud University Nijmegen, 2013. 